\title{
Study on High Load Can Enhance the Capacity of the Consumptive Way
}

\author{
Jing-jing Zheng ${ }^{\mathrm{a}}$, Yong Yang ${ }^{\mathrm{b}}$, Pei-dong $\mathrm{Du}^{\mathrm{c}}$, Jing Peng ${ }^{\mathrm{d}}$ \\ Gansu Electric Power Research Institute, Guangzhou 510000, China; \\ ajingjing110@163.com, 'yy8801@126.com, ' dpdong@126.com, ${ }^{\mathrm{b}}$ pengjing19871028@163.com
}

Keywords: New energy; load; given and high load.

\begin{abstract}
With the continuous expansion of the scale of new energy, new energy to the grid are also increasingly prominent, mainly reflected in: on the one hand, due to the new energy base in Jiuquan province from the load center, and the Hexi area load is small, large-scale new energy to local consumption; on the other hand, due to the randomness of the new energy, intermittent and semi controllable characteristics, when the operation of the new energy grid, must be made by the conventional power supply for its active power compensation, to ensure reliable power supply to the load security. High energy consuming industries as a kind of advanced technology, environmental protection industry energy utilization rate is high, the electricity load is large, and therefore, high key development in Hexi area energy industry is an important way of wind power.
\end{abstract}

\section{Introduction}

Hexi Corridor of Gansu Province is rich in wind power resource, most areas in solar radiation are rich in resources and relatively rich area, Gansu has the development of new energy unique comprehensive advantages of. In 2013, Gansu Province of new energy installed capacity exceeded 10 million kilowatts, photovoltaic, wind power installed capacity reached 429.84 million kilowatts and 702.81 million kilowatts, respectively, ranking the No. 1 and No. 3.

According to the general planning of Jiuquan new energy base, to 2015 in Jiuquan wind, photovoltaic installed capacity will reach 12.71 million $\mathrm{kW}$, by 2020 installed capacity will reach 20 million $\mathrm{kW}$, after 2020, the installed capacity is expanded to more than 30 million $\mathrm{kW}$, Jiuquan and Hexi corridor will become the world's largest new energy base. High load can load to enhance the local absorptive capacity problem is particularly prominent.

\section{General situation and existing problems of Jiuquan wind power base}

When conventional energy peaking capacity can't meet the Hexi area in the development of new energy, the high load to stabilize the fluctuation of wind power, the large-scale new energy generation can place consumptive, thus cutting the peak and filling valley of. However, different high load can be load connected to the power grid, will on the grid produced different effects, which would have great influence on electric power supply and demand balance and power network loss ${ }^{[1]}$.

\section{High load impact on load wind power fluctuation}

\subsection{High energy load on the wind power consumptive impact.}

Wind power construction at the same time, we must simultaneously consider the consumptive problem; consumptive channels have local consumptive and delivery of two ways. In Hexi Corridor Zhangye, Jinchang, Wuwei City, wind power installed capacity is small and local electrical load is large, wind power can consumptive. In the Hexi Corridor, the wind power mainly concentrated in Jiuquan area, and area of Jiuquan wind power away from the province's electricity load centers, and the Jiayuguan and Jiuquan wind power consumptive ability is very limited, a lot of wind power can't consumptive $^{[2]}$. The Jiuquan and Hexi wind power through double circuit $750 \mathrm{kV}$ transmission line to transported outside the province, considering the need the stable operation of the system, actual 
transmission capacity of about 300 million KW, the large amount of wind power is restricted output, the impact of the enthusiasm for the development of wind power.

Only a small contribution to the local minimum not only save place due to the Jiuquan wind power installed capacity is large, the delivery capacity is limited, and the delivery channel construction period is longer, local consumptive is the need to focus on the way ${ }^{[3]}$. Wind power consumptive power grid construction investment, and the transmission loss. So, the development of electricity load, local consumptive wind power can be directly to the local wind power resources advantage into economic advantage, for the wind power industry sustainable development to provide protection. The development of small and medium enterprises, electricity load is small, the consumptive a large number of rich wind power, therefore, focus on the development of power load is bigger than that of the high energy industry to accommodating wind power will be more effective ${ }^{[4]}$.

Jiayuguan and Jiuquan to accelerate the development of modern high load energy industry has the following advantages:

First, species and reserves of mineral resources are very rich, with white marble only Jiuquan Guazhou County silica resources reserves amounted to 10 billion cubic meters, Liuyuan region silica resources and prospective reserves of more than 10 million tons;

Secondly, modern development and high load can industry place consumptive surplus power and energy output for the output of the products is energy advantages into economic advantages in an effective way.

Third, speed up the development of modern high energy carriers industry is to implement the country to undertake industrial transfer policy actions; the fourth, high drug industry is a traditional advantage industry of Gansu Province, high load can be the total assets of the industry, industrial added value; total profits more than $80 \%$ of the province's total industrial output to.

Therefore, to accelerate the development of modern high load energy industry, expand the scale of the industry, enhance the total industrial economy, promote industrial upgrading, is an important measure to promote the development of industry in Gansu Province.

\subsection{Study on the influence of high load energy on wind power fluctuation.}

Because the device such as boiler, steam turbine limited by alternating stress, slower adjustment, the general condensing units per minute can be adjusted only about $1 \%$ of the installed capacity, for cogeneration unit, the minimum output and adjusts the speed but also restricted by heating. Thus it can be seen that the conventional power ability to participate in peak shaving cannot satisfy the development of wind power of hexi area, need to study wind "direct" and "in situ transformation, development to adapt to the new energy power generation output characteristic of high-tech, high added value to industry ${ }^{[5]-[7]}$.

Existing research results show that the chlor alkali and electrolytic aluminum and other high load production load can better adapt to the characteristics of wind power, voltage or current fluctuations in the production process, only affect the product yield, does not affect the quality of product and process; dual power supply, power grid automatically add wind power, to ensure the stable production of electrolytic cell. Therefore, it can be in power grid peak capacity is insufficient, by controlling the high load can load to stabilize the fluctuation of wind power, so as to cut a peak to fill valley ${ }^{[8]}$.

\section{Study on control scheme of high energy wind power fluctuation}

The high adaptability of load of wind power fluctuation can be learned, in high load to load in the production process, the fluctuation of voltage or current does not affect the product quality. Therefore, when the grid adjustment ability is not enough to deal with the wind power fluctuations can through short time interrupt high load can load to smoothing the wind power fluctuation. High load can load smoothing the wind power fluctuation control scheme to solve the main problem is in the premise of ensuring the safe and stable operation of the system, by controlling the high load can load switching to stabilize the fluctuation of wind power, to maximize the use of wind power. The control objectives include: 
(1) ensure a reliable and stable operation;

(2) improve wind power utilization rate;

(3) when high load can load the interrupt and interrupt number reaches the upper limit, to take necessary measures to ensure reliable power to the load;

(4) to ensure that the system fault can be fast enough to isolate the fault, reduce the power load and the impact of the power system operation.

High load can be load smoothing the wind power fluctuation control of grid control purpose, but the control method is different. Through the high load control method to stabilize the fluctuation of wind power is from the full use of wind power angle of and maximize the reduce of wind farm output limit, as far as possible to make wind power. Therefore, for the case of high load control load smoothing the wind power fluctuation, by removing or access load achieves greatly active power regulation in order to adapt to the fluctuation of wind power is the main means of control. That is to say, the active power control is not the main target of wind farm, but high load can load.

Combined with high load operation characteristics and wind power forecasting research results, to wind power utilization maximization as the goal, made by high load can be load smoothing the wind power fluctuation of three control strategies, as follows:

(1) predictive control

Based on the short term forecasting curve and the load forecasting curve, the wind farm output is defined as a negative load, which is superimposed on the original load, and the equivalent load curve is formed;

(2) conventional power control

By conventional power smoothing the wind power fluctuation control strategy includes:

Step 1: according to the equivalent load curve, considering fire group of motor operation and unit constraints based on, hydro units and thermal power generation plan arrangements;

Step 2: according to the thermal power generation plan and rated capacity determined reserve capacity of thermal power units, to leave a part of reserve capacity for wind power peak and another part as a reserve capacity margin;

Step 3: when the wind wave motion, according to thermal power units for the size of the backup capacity of wind power peak, through the adjustment of thermal power units of output power to smoothing the wind power fluctuation;;

(3) high load energy load control

The high load smoothing the wind power fluctuation control strategy includes:

Step 1: when the thermal power peaking capacity can't meet the wind power fluctuation, through packet switching of high load to stabilize the fluctuation of wind power;

Step 2: when the actual wind farm output and output prediction, there is a deviation, the thermal power units leave some reserve capacity margin to regulate the output power, in order to achieve the power balance of the whole system.

\section{Research on coordinated control strategy of new energy and high load energy load}

\subsection{Research on Hierarchical Coordination Control Strategy of new energy and high load energy load.}

For research on new energy and high load can load layered coordinated control method. First of all, we need will power according to the voltage level is divided into a number of layers, on each layer of the sub grid established hierarchical model, and according to the topology circuit of transmission capacity and load calculated in each sub grid should be access to the high load capacity and location. When a node load fluctuation, the use of in the same level of new energy power plant with high drug load regulation and absorptive capacity, the power of the nodes and the hierarchy of the maintained at a stable level.

(1) method for calculating load capacity and position of high load capacity 
To calculated after stratification of sub grid access of high load, the size and position of the load, and needs to find out the layer at all levels, each node can access high load can load value. And according to the transmission line capacity of load distribution.

For an electrical network, it should satisfy the following properties: the load of the upper node is equal to the sum of the load of all the nodes connected to it and the load of all the arcs between them.

The calculation process can calculate the load of each node to the value. When the load of a node due to the fluctuation of the, need to plant and high load regulation. The layer in new energy and load to achieve the balance of supply and demand. This makes the power grid is relatively stable.

(2) coordinated control strategy of new energy and high load energy load

The following are the grid hierarchical control process:

1) the Hexi area power grid according to the voltage level is divided into different levels.

2) hierarchical model establish sub grid.

3) calculation of the topological structure of each node can access the load value. If the high load capacity is larger than the access node for access load value, high load can't be the construction of this node. When high load capacity is less than the access node can access the load value, if the high load of the original node is larger than the line between the node and the corresponding node maximum transmission capacity and load, also can't build at this point

4) The size and location of the node can access the load capacity for high load level.

5) When a node load fluctuation, the use of in the same level of new energy power plant with high drug load regulation and absorptive capacity, the power of the nodes and the hierarchy of the maintained at a stable level.

\subsection{Research on coordinated control strategy of new energy and high load energy load}

In order to study new energy and high load can subarea load coordinated control method, first of all to according to the access mode of the new energy where the geographical location and wind farm, the clustering method to partition the grid is then calculated within each region of the high load can load access capacity and location, in order to achieve a balance of power in the region. When the region, the regional load due to the fluctuation of the, according to the need of new energy and high load of regulation, the power supply and demand to maintain a basic balance.

(1) research on coordinated control strategy of new energy and high load energy load.

In the area corresponding to the access to high load, when regional load fluctuations, you can adjust region of the power plant and the high load can load, phase equilibrium of the generated power and the consumptive power. When the new energy from power plants, power fluctuation, regulation of high load to load the consumptive power, makes the system partition in the basic balance of supply and demand.

Adjusts supply. By the end of the output power of power plants can make two area for the power balance of power plant load. Interconnected power grid tie line power optimization model and the power grid in the new energy power plants have close contact on the contact line of actual contribution rate. Optimal allocation of grid in the new energy power plant power generation scheme, a reasonable allocation of power plant and tie line power correlation factor, coordinated power output can be realized reduce restricted power statute tie line active power balance.

(2) strategy of coordinated control of new energy and high load energy load

Partition control strategy process:

1) according to the wind farm in the Hexi area set access point grid division;

2) the electrical distance between the power plant for K-means clustering index, the grid re partition to obtain compact and independent regions. And the contact line between regions into areas where the equivalent load;

3) the total power output of the calculation area access in different new energy power plant and total load value, then calculate the high load value required to access;

4) within the area of load fluctuation, can adjust the area of new energy power plants and high load. When the new energy power from the power plant wave, adjustable high load power load consumption, making the supply and demand system partition in the basic balance. 
5) coordinated control between regions, when a region load fluctuation, the sensitivity coefficient is defined in this section of Power plant for inter-regional power conditioning, so that the stability of the system to maintain.

\section{Conclusion}

When conventional energy peaking capacity can't meet the Hexi area in the development of new energy, the high load to stabilize the fluctuation of wind power, the large-scale new energy generation can place consumptive, thus cutting the peak and filling valley of. However, different high load can be load connected to the power grid, will on the grid produced different effects, which would have great influence on electric power supply and demand balance and power network loss.

\section{References}

[1]. Wang Ningbo. The challenge for the jiuquan never kw wind power base and response [J]. Power grid and clean energy, 2009, 25 (7) : 43-47.(In Chinese)

[2]. Bowwah lau, dong-rong wang, Ming zeng. From the demand side management to demand side response [J]. Electric power demand side management, 2005, 8 (5): 10 to 13.(In Chinese)

[3]. The gansu province electric power company. 2012 annual operation mode [R]. Lanzhou: gansu province electric power company, 2012.(In Chinese)

[4]. Zhang Qian Li Hu, takamatsu. Wind power research on the influence of load and its reasonable use patterns [J]. Journal of southern power grid technology, 2010, 4 (6): 19 to 20.(In Chinese)

[5]. Guoping zhang, Zhou Youxue. Energy power saving measures study [J]. China electric power, 2012, 2 (5) : 75-77.(In Chinese)

[6]. Chang chin, xi-fan wang, jian-xue wang, etc. Under the electricity market demand response research review [J]. Automation of electric power systems, 2008, 32 (3) : 97-99.(In Chinese)

[7]. Hai-ying Lin, jian-rong li, XuanJuQin, etc. The steel industry load characteristic and the DSM potential analysis [J]. Journal of east China power, 2005 (11) : 831-834.(In Chinese)

[8]. Qin Rui, liu haiyan, Yang ping, etc. Large scale wind power out ability influence factors analysis [J]. Electric power construction, 2013 (6): 36 to 40.(In Chinese) 